\title{
STUDY OF SENSITIVITY PATTERN OF METRONIDAZOLE ON AXENIC CULTURE OF ENTAMOEBA HISTOLYTICA IN VITRO
}

\author{
SARKER SK ${ }^{1}$, MONDAL D ${ }^{2}$, SIDDIQUE MA ${ }^{3}$
}

\begin{abstract}
Objective: To evaluate the sensitivity pattern of metronidazole against Entamoeba histolytica.

Design: It was an experimental study.

Settings: The study was carried out in Parasitology Laboratory, ICDDR,B Dhaka from January 2007 to June 2007.

Subjects: Entamoeba histolytica (Strain: MS 27 5030)

Main outcome measure: Minimum inhibitory concentration (MIC).

Results: In this study MIC of metronidazole was $<0.08 \mathrm{mg} / \mathrm{ml}$.

Conclusion: The result suggests that metronidazole is still the standard drug for treatment of amoebiasis
\end{abstract}

Key words: Axenic culture, Entamoeba histolytica, Metronidazole.

J Dhaka Med Coll. 2010; 19(1) : 65-66.

\section{Introduction:}

In 1969, WHO defined amoebiasis, a condition in which a patient harbouring the organism Entamoeba histolytica in the bowe ${ }^{1}$. Culture of $E$. histolytica is a long and laborious process. There are three basic types of culture systems of $E$. histolytica: xenic, in which the parasite is grown in the presence of an undefined flora; monoxenic, in which the parasite is grown in the presence of a single additional species; and axenic in which the parasite is grown in the absence of any other metabolizing cells ${ }^{2}$.

Axenic cultivation of $E$. histolytica was first accomplished by Diamond in 1961. Diphasic medium was first introduced for cultivation of organism. In the course of developing media, TP-S-1, TYI-S-33 and YI-S media were also introduced for cultivation of organism. LYI-S-2 medium, containing liver digest, yeast extract, iron and serum was introduced few years for cultivation of $E$. histolytica ${ }^{2}$.
Metronidazole is the drug of choice for the treatment of amoebiasis and has been used in clinical practice for many years. In asymptomatic individuals, inappropriate usage of drugs could lead to drug resistance ${ }^{3}$. Drug resistance in E. histolytica is uncommon but differences in drug susceptibility between different isolates have been reported and resistance of metronidazole may develop in future $^{4}$. So there is a need of continuous screening to observe the resistant pattern of metronidazole against E. histolytica.

Therefore, the present study was conducted to observe the sensitivity pattern of metronidazole on axenic culture of E. histolytica.

\section{Methodology:}

Antiamoebic test was done at the Parasitology Laboratory, ICDDR,B Dhaka. Metronidazole was collected from Beximco Pharmaceuticals Ltd. Then stock solution of $1 \mathrm{mg} / \mathrm{ml}$ was prepared.

1. Assistant Professor, Department of Pharmacology \& Therapeutics, Dhaka Medical College, Dhaka.

2. Associate Scientist, Parasitology Laboratory, ICDDR,B, Dhaka.

3. Research Officer, Parasitology Laboratory, ICDDR,B, Dhaka.

Correspondence: Dr. Sujit Kumar Sarker 
Axenic culture of E. histolytica (Strain: MS 27 5030) and LYI-S-2 medium was kindly provided by Dr. Rashidul Haque, Head of Parasitology Laboratory, ICDDR,B Dhaka. Serial dilutions of stock solution in culture media were made from $200 \mathrm{mg}$ to $0.8 \mathrm{mg} / \mathrm{ml}$. Several trials were conducted for the study. Each trial included blank (only culture medium), control (axenic culture) and test (axenic culture and metronidazole). The 24 well culture plates were incubated at $37^{\circ} \mathrm{C}$ for 48 hours. After incubation viable trophozoites of $E$. histolytica were counted with the aid of Neubauer's cell of Haemocytometer.

\section{Results:}

The MIC is defined as the lowest concentration of a drug that will inhibit the visible growth of an organism after overnight incubation ${ }^{5}$. In this study MIC was defined as the lowest concentration of metronidazole that inhibited the growth of E. histolytica after 48 hours incubation.

Figure-I shows the results of metronidazole study. In this study concentrations of metronidazole were $200,100,50,25,12.5,6.25$, $3.12,1.6,0.8 \mathrm{mg} / \mathrm{ml}$. In blank group there was no growth of organism. In control group there was increased count of organism compared to initial count. But in test group growth of $E$. histolytica was inhibited by all the concentrations of metronidazole. Therefore, minimum inhibitory concentration (MIC) was $<0.8 \mathrm{mg} / \mathrm{ml}(\mathrm{P}<0.001)$. There is also a linear trend between percentage inhibition of $E$. histolytica and concentration of metronidazole.

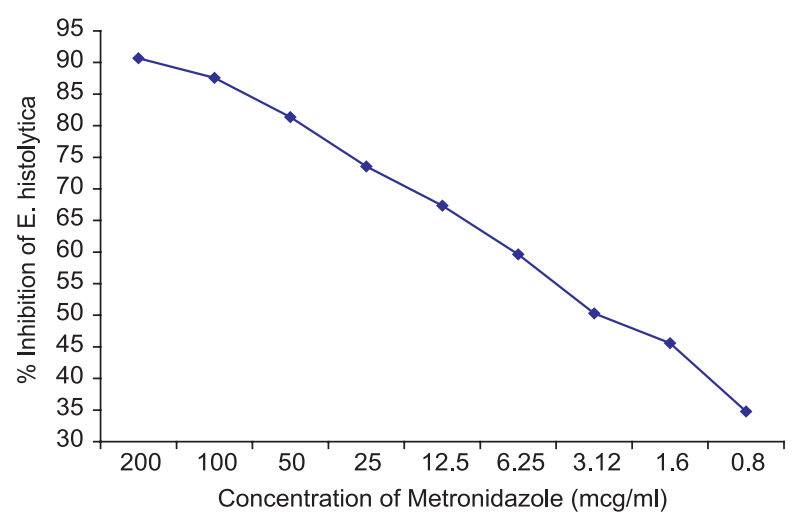

Fig.-1: Growth inhibition of organism in presence of different concentrations of metronidazole

\section{Discussion:}

In the present study axenic culture of $E$. histolytica were subjected to susceptibility test against metronidazole. Previous study reported mean $\mathrm{IC}_{50}$ value of metronidazole was $13.2 \mu \mathrm{m}^{4}$ and another study reported minimum inhibitory concentration (MIC) of metronidazole ranging from $12.5-25 \mu \mathrm{m}^{6}$. To understand the magnitude of drug resistance one study reported that the mean 50 percent inhibitory concentration $\left(\mathrm{IC}_{50}\right)$ value to metronidazole was $18.47 \mu \mathrm{m}$ for the most susceptible isolates of $E$. histolytica and $>30 \mathrm{~mm}$ value was the cut-off value for resistance ${ }^{7}$. Although drug resistance is not a serious problem till today but it may naturally develop in future. The results of the present study are in consistence with findings of the previous study but difference in sensitivity pattern which may be due to difference in culture of $E$. histolytica, strain of organism, culture medium or raw materials of metronidazole.

\section{Conclusion:}

In conclusion, the present study suggests that metronidazole is still sensitive to E. histolytica but continuous study is needed to explore the resistance pattern of metronidazole.

\section{Acknowledgement:}

The authors are grateful to the authority Parasitology Laboratory, ICDDR,B, Dhaka for providing laboratory facilities.

\section{References:}

1. Keene AT, Harris A, Phillipson JD, Warhurst DC. In vitro amoebicidal testing of natural products; Part1. Methodology. Planta Med. 1986; (4): 278-85.

2. Clark CG and Diamond LS. Methods for cultivation of luminal parasitic protists of clincial importance. Clin Microbiol Rev. 2002; 15(3): 329-41.

3. Bansal D, Sehgal R, Chawla Y, Malla N, Mahajan RC. Multidrug resistance in amoebiasis patient. Indian J Med Res. 2006;124:189-94.

4. Bansal D, Sehgal R, Chawla Y, Mahajan RC Malla N. In vitro activity of antiamoebic drugs against clinical isolates of Entamoeba histolytica and Entamoeba dispar. Ann Clin Microbiol. 2004; 3: 27-33.

5. Andrews JM. Determination of minimum inhibitory concentrations. J Antimicrob Chemother. 2001; 48 (suppl 1):5-16.

6. Upcroft P, Upcroft JA. Drug targets and mechanism of resistance in the anaerobic protozoa. Clin Microbiol Rev. 2001; 14: 150-64.

7. Adagu IS, Nolder D, Warhurst DC, Rossignol JF. In vitro activity of nitazoxanide and related compounds against isolates of Giardia intestinalis, Entamoeba histolytica, Trichomonas vaginalis. J Antimicrob Chemother. 2002; 43: 103-11. 